\title{
Put patients first and give the money back
}

\author{
Fiona Godlee editor in chief, The BMJ
}

John Townsend wants to say thank you before he dies. "All this bullshit about the NHS being rubbish is not true," he writes this week (doi:10.1136/bmj.h4706). "I've been treated so wonderfully that you wouldn't believe it." Although his cancer returned, his satisfaction with the promptness of his care suggests that his GP used the fast track referral pathway for suspected cancer. And we now have evidence that this pathway works. Henrik Møller and colleagues found better outcomes among patients whose doctors made more use of fast track referral (doi:10.1136/bmj.h5102). On this basis, failing to fast track patients with suspected cancer looks like poor practice.

How then would secondary care services cope if all GPs made full use of fast track referral, especially at the new lower referral thresholds recommended by NICE in its recent guidance (doi:10. 1136/bmj.h3044)? Given the dire financial forecast for England's NHS (doi:10.1136/bmj.h5430), few can doubt that more money is urgently needed. Without it, care of patients will suffer, and the current crisis could be used to erode the public's faith in an NHS that is free at the point of care. But what of the longer term? Shouldn't healthcare learn to live within its means? This was Don Berwick's theme at the joint BMJ and Institute for Healthcare Improvement's Asia Quality Forum last month (http://internationalforumasia.bmj.com). More money spent on healthcare means less money for other things that societies need to flourish: welfare, education, art, culture, sport, the environment. David Taylor-Robinson and colleagues press this point in their editorial on the effects on children's development of welfare cuts (doi:10.1136/bmj.h5330). There must be a point, said Berwick, at which healthcare cost, as a proportion of gross domestic product, levels off and even contracts. His mantra is "put patients first and give the money back." The "triple aim" created by Berwick and colleagues at the Institute for Healthcare Improvement is for better care, improved population health, and lower cost.

One key to lowering costs (as well as reducing harms) is to do less of what doesn't work. The list of questionable or low value interventions is already long, thanks to various initiatives around the world: Choosing Wisely, Prudent Healthcare, and "slow medicine." This week we highlight two more interventions for the list: screening healthy people for coronary heart disease with coronary computed tomography (doi:10.1136/bmj.h4926) and treating subclinical hypothyroidism in pregnancy (doi:10.1136/ bmj.h4726).

As for improving population health, there can be nothing more important, after tobacco control, than tackling the public health catastrophe caused by the adoption worldwide of rich countries' diet. The global prevalence of obesity and overweight is rising sharply, concluded the World Obesity Federation last week (www.theguardian.com/society/2015/oct/09/obesitys-globalspread-un-goals-diet-related-diseases-fail), largely as a result of consumption of sugary soft drinks and snacks aggressively promoted by multinational companies. What will governments do about this? In the UK a report commissioned from Public Health England is sitting on Jeremy Hunt's desk. Sarah Wollaston, chair of the health select committee, says she needs to see it (doi:10.1136/bmj.h5469). So do we all. PHE's chairman, Duncan Selbie, should assert his independence from government and release the report.

Cite this as: BMJ 2015;351:h5489

๑ BMJ Publishing Group Ltd 2015 